\title{
Application of QLM-Rational Legendre collocation method towards Eyring-Powell fluid model
}

https://doi.org/10.1515/nleng-2018-0005

Received January 6, 2018; revised June 1, 2018; accepted June 7, 2018.

\begin{abstract}
In this paper, a spectral method based on the rational Legendre functions is discussed to approximate the solution of the boundary layer flow of an Eyring-Powell non-Newtonian fluid over a stretching sheet. At first, because of the model is a nonlinear ordinary differential equation, we construct a sequence of linear ordinary differential equations (ODEs) by using the quasilinearization method (QLM). By applying QLM on the ODEs at each iteration, the equations convert to a system of linear algebraic equations. The present results have shown the high attention and convergence of our method, and the residual error of the present results are very small. In addition, the effect of the Eyring-Powell fluid material parameters is explained.
\end{abstract}

Keywords: Boundary Layer Flow; Eyring-Powell NonNewtonian Fluid; Rational Legendre Functions; Quasilinearization Method

MSC: 76A05; 74S25; 76D05; 76M55; 34B40

\section{Introduction}

Considering the engineering and technological applications one of the most increasing interest for the last few decades the flows of non-Newtonian fluids. Continuous stretching of plastic films, artificial fibers, metal extrusion, glass blowing are some important applications in industries [1]. In length manufacture of these sheets, the melt

\footnotetext{
*Corresponding Author: Kourosh Parand, Department of Computer Sciences, Shahid Beheshti University, G.C. Tehran, Iran Department of Cognitive Modeling, Institute for Cognitive and Brain Sciences, Shahid Beheshti University, G.C. Tehran, Iran, E-mail: k_parand@sbu.ac.ir

Fatemeh Mirahmadian, Department of Computer Sciences, Shahid Beheshti University, G.C. Tehran, Iran, E-mail:

f.mirahmadian@mail.sbu.ac.ir

Mehdi Delkhosh, Department of Mathematics and Computer Science, Islamic Azad University, Bardaskan Branch, Bardaskan, Iran, E-mail: mehdidelkhosh@yahoo.com
}

issues from a slit and is subsequently stretched to achieve the desired thickness. The mechanical properties of the final product strongly depend on the stretching and cooling rates in the process [2,3]. Industrial applications, such as combustion systems, metallurgy, nuclear reactor safety, solar collector and chemical engineering are some of applications of stretched flows in different engineering and industrial applications [4]. Crane [5] at first studied the steady boundary layer flow of the incompressible flow. There are two types of fluid whose flows are investigated: Newtonian and non-Newtonian. The Newtonian law is the only unique relationship existing in the paper [6]. Significant contributions to the study of non-Newtonian fluid models with a diversity of rheological properties have been made by Harris [7] and Bird et al. [8]. Mathematical systems for non-Newtonian fluids are of higher order and intricate in comparison to the Newtonian fluids. The nonNewtonian fluid models differ in their intricacy and capability to catch different physical phenomena. Different models are used to represent different characteristics of the non-Newtonian fluids because no single model can capture all the features of the non-Newtonian fluids complexities [6, 9]. Non-Newtonian fluids gained much attention due to their various applications in physiological and industrial processes [10] such as coal water, jellies, toothpaste, ketchup, and food products [6]. The analytic/numerical studies of non-Newtonian fluids models show exciting challenges to mathematicians, physicists, numerical simulists and modelers [11]. One of the most important models for non-Newtonian fluids is the EyringPowell model. Eyring-Powell model deduced from kinetic theory, can be reduced to the Newtonian behavior for low and high shear rates. Takhar et al. [12] studied the MHD flow and mass transfer in a viscous fluid over a stretching surface. In addition, the flow and heat transfer of the Powell-Eyring fluid in the boundary layers on an exponentially stretching continuous permeable surface was studied by Megahed [13]. Newly, researches on the Lie group analysis in the field of fluid science to encountered heat and mass transfer effects simultaneously have been done [14] and also Ellahi et al. [15] worked on the MHD flow of an Eyring-Powell fluid between parallel heated plates by pseudo-spectral collocation method. Also, the studies 
have been done which aims to investigate the effects of magnetic field and anisotropic slip on the flow of EyringPowell fluid and heat transfer over an infinite rotating disk [16], the research on the deals with the fully-developed two-layer Eyring-Powell fluid in a vertical channel divided into two equal regions have been done [17], and the double diffusive Darcian convection flow of Eyring-Powell fluid from a cone embedded in a homogeneous porous medium with the effects of Soret and Dufour has been studied [18]. Nonlinear problems are important in fluid mechanics and heat transfer. These nonlinear equations should be solved using numerical methods as most of them do not have analytical solutions. To approximate linear and nonlinear ordinary differential equations (ODEs) The collocation approaches based on variant functions, such as radial basis functions(RBF), Laguerre functions, etc., have been used in various sciences like astronomy, physics, and biology [19]. The rational Legendre functions are the most useful orthogonal functions, which are very efficient for solving different problems in the finite and semi-infinite intervals as they can approximate the solutions with high accuracy [20-23].

In this paper, we want to study the boundary layer flow of an Eyring-Powell fluid over a stretching sheet in an unbounded domain by the rational Legendre functions collocation method with the QLM to find an approximate solution for the problem. In the continue, this paper are organized as follows: in section 2, we express the mathematical model and in section 3, preliminaries are explained. Section 4 contains the numerical solution of the problem. Afterward, numerical results are explained in section 5 . The conclusion is presented in the last section.

\section{Mathematical model of the problem}

For the incompressible fluid obeying Eyring-Powell model, the equation of continuity and the $x$-momentum equation can be simplified using the boundary layer approximation as (see Fig. 1) [2]:

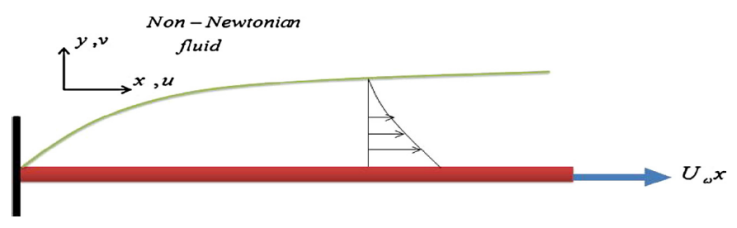

Fig. 1: Velocity boundary layers on a stretching sheet.

$$
\begin{aligned}
\frac{\partial u}{\partial x}+\frac{\partial v}{\partial y} & =0 \\
u \frac{\partial u}{\partial x}+v \frac{\partial u}{\partial y} & =\left(\alpha+\frac{1}{\rho \beta H}\right) \frac{\partial^{2} u}{\partial y^{2}}-\frac{1}{2 \rho \beta H^{3}}\left(\frac{\partial u}{\partial y}\right)^{2} \frac{\partial^{2} u}{\partial y^{2}}
\end{aligned}
$$

By following boundary conditions:

$$
\begin{cases}u=U_{\omega} x=b x, v=0 & \text { at } y=0, \\ u \rightarrow 0 & \text { at } y \rightarrow \infty .\end{cases}
$$

We proceed for solutions through stream function satisfying bellow:

$$
u=\frac{\partial \psi}{\partial y}, \quad v=-\frac{\partial \psi}{\partial x}
$$

and

$$
\psi=(b \alpha)^{\frac{1}{2}} x g(\eta) .
$$

In addition, $\eta$ is defined as

$$
\eta=\left(\frac{b}{\alpha}\right)^{\frac{1}{2}} y
$$

The following equation is obtained using the above equations:

$$
(1+\varepsilon) \frac{d^{3} g}{d \eta^{3}}-\varepsilon \delta\left(\frac{d^{2} g}{d \eta^{2}}\right)^{2} \frac{d^{3} g}{d \eta^{3}}-\left(\frac{d g}{d \eta}\right)^{2}+g \frac{d^{2} g}{d \eta^{2}}=0,
$$

where $\varepsilon$ and $\delta$, the material fluid parameters, are defined as:

$$
\varepsilon=\frac{1}{\mu \beta H}, \quad \delta=\frac{b^{3} x^{2}}{2 H^{2} \alpha} .
$$

The boundary conditions for Eq. (6) are defined as follows:

$$
\left\{\begin{array}{l}
g(0)=0, \\
g^{\prime}(0)=1, \\
g^{\prime}(\eta)=0 \quad \eta \rightarrow \infty
\end{array}\right.
$$

In the above model, $\mu$ is the dynamic viscosity of the fluid, $\rho$ is the fluid density, $\alpha=\frac{\mu}{\rho}$ is the kinematic viscosity of the fluid, $H$ and $\beta$ are the material parameters, $b$ is the linear stretching velocity, $U_{\omega}$ is the stretching velocity, and $\varepsilon$ and $\delta$ are the non-dimensional fluid parameters.

In the next section, the solution to Eq. (6) will be approximated by the rational Legendre functions collocation method.

\section{Preliminaries}

In this section, the Legendre polynomials, the rational Legendre functions, and the QLM method are explained. 


\subsection{Rational Legendre functions}

Legendre polynomials are orthogonal in the interval $[-1,1]$ with respect to the weight function $w(x)=1$ and can be specified by the help of the following recurrence formula [24]:

$$
\begin{gathered}
P_{0}(x)=1, P_{1}(x)=x, \\
P_{n+1}(x)=\left(\frac{2 n+1}{n+1}\right) x P_{n}(x)-\left(\frac{n}{n+1}\right) P_{n-1}(x), \quad n \geq 1 .
\end{gathered}
$$

Legendre polynomials are orthogonal on the interval $[-1,1]$ :

$$
\int_{-1}^{1} P_{n}(x) P_{m}(x) d x=\frac{2}{2 n+1} \delta_{m n} .
$$

The new basis functions, denoted by $R P_{n}(x)$, are explained as:

$$
R P_{n}(x)=P_{n}\left(\frac{x-L}{x+L}\right),
$$

where the constant parameter $L$ sets the length scale of the mapping. Boyd in page 377 of his famous book [25] offered guidelines for optimizing the map parameter $L$ for rational Chebyshev functions: "The experimental trial-anderror method", that is also useful for rational Legendre functions.

The $n$th eigenfunction of the singular Sturm-Liouville problem is $R P_{n}(x)$ :

$$
\begin{gathered}
\frac{(x+L)^{2}}{L}\left(x\left(R P_{n}^{\prime}(x)\right)^{\prime}+n(n+1) R P_{n}(x)=0,\right. \\
x \in[0, \infty], n=0,1, \cdots
\end{gathered}
$$

and by Eq. (8), they satisfy in the following recurrence relation:

$$
\begin{gathered}
R P_{0}(x)=1, \quad R P_{1}(x)=\frac{x-L}{x+L}, \\
R P_{n+1}(x)=\left(\frac{2 n+1}{n+1}\right)\left(\frac{x-L}{x+L}\right) R P_{n}(x)-\left(\frac{n}{n+1}\right) R P_{n-1}(x), \quad n \geq 1 .
\end{gathered}
$$

\subsection{The quasilinearization method}

Quasilinearization method (QLM) is a technique to find a solution for nonlinear $\mathrm{n}$-th order differential equation, ordinary or partial, in $\mathrm{N}$ dimensions as a limit of a sequence of linear differential equations [26]. Bellman and Kalaba expanded this method at first, which is based on the Newton-Raphson method [27]. Also, the best property of this approach is its quadratic convergence [28-30].

We suppose to have a nonlinear differential equation on domain $[0, \infty)$ as follows:

$$
\frac{d^{3} y}{d \eta^{3}}=G\left(y^{\prime \prime}(\eta), y^{\prime}(\eta), y(\eta), \eta\right),
$$

and the boundary conditions:

$$
y(0)=A, \quad y^{\prime}(0)=B, \quad y^{\prime}(\infty)=C,
$$

where $\mathrm{A}, \mathrm{B}, \mathrm{C}$ are real constants.

The solution of Eq. (9) determines by using the QLM as a solution of the linear ODE at $(i+1)$-th iterations as follows:

$$
\begin{aligned}
\frac{d^{3} y_{i+1}}{d \eta^{3}}= & G\left(y_{i}^{\prime \prime}, y_{i}^{\prime}, y_{i}, \eta\right)+\left(y_{i+1}-y_{i}\right) G_{y}\left(y_{i}^{\prime \prime}, y_{i}^{\prime}, y_{i}, \eta\right) \\
& +\left(y_{i+1}^{\prime}-y_{i}^{\prime}\right) G_{y^{\prime}}\left(y_{i}^{\prime \prime}, y_{i}^{\prime}, y_{i}, \eta\right) \\
& +\left(y_{i+1}^{\prime \prime}-y_{i}^{\prime \prime}\right) G_{y^{\prime \prime}}\left(y_{i}^{\prime \prime}, y_{i}^{\prime}, y_{i}, \eta\right),
\end{aligned}
$$

and the boundary conditions

$$
y_{i+1}(0)=A, \quad y_{i+1}^{\prime}(0)=B, \quad y_{i+1}^{\prime}(\infty)=C,
$$

where the functions

$$
G_{y}=\frac{\partial G}{\partial y}, \quad G_{y^{\prime}}=\frac{\partial G}{\partial y^{\prime}}, \quad G_{y^{\prime \prime}}=\frac{\partial G}{\partial y^{\prime \prime}},
$$

are functional derivatives of the function $G\left(y_{n}^{\prime \prime}, y_{n}^{\prime}, y_{n}, \eta\right)$, and the prime explains the ordinary derivative with respect to $\eta$, and $n=0,1, \cdots$.

The QLM iteration needs an initialization or initial guess $y_{0}(\eta)$ that it is chosen from physical and mathematical considerations or the boundary conditions in the equation. Furthermore, Mandelzweig and Tabakin in their paper [26] have fully studied the convergence of the QLM and they showed that QLM is convergent from rate $O\left(h^{2}\right)$, and also Canuto et al. [31] have proved the stability and convergence analysis of spectral methods.

\section{Application of the method}

In this section, we solved the equation (6) by QLM based on rational Legendre collocation method.

First, by using QLM, the solution of Eq. (6) specifies the $(i+1)$-th iterative approximation $g_{i+1}(\eta)$ as a solution for the following linear $\operatorname{ODE}(i=0,1, \cdots)$ :

$$
\begin{aligned}
g_{i+1}^{\prime \prime \prime}(\eta)= & G+\left(g_{i+1}(\eta)-g_{i}(\eta)\right) \frac{d G}{d g_{i}(\eta)} \\
& +\left(g_{i+1}^{\prime}(\eta)-g_{i}^{\prime}(\eta)\right) \frac{d G}{d g_{i}^{\prime}(\eta)} \\
& +\left(g_{i+1}^{\prime \prime}(\eta)-g_{i}^{\prime \prime}(\eta)\right) \frac{d G}{d g_{i}^{\prime \prime}(\eta)},
\end{aligned}
$$

in which $G=\frac{g_{i}^{\prime 2}(\eta)-g_{i}(\eta) g_{i}^{\prime \prime}(\eta)}{\varepsilon+1-\varepsilon \delta g_{i}^{\prime \prime 2}(\eta)}$.

Furthermore, the boundary conditions are defined as follows:

$$
\left\{\begin{array}{l}
g_{i+1}(0)=0, \\
g_{i+1}^{\prime}(0)=1, \\
g_{i+1}^{\prime}(\infty)=0
\end{array}\right.
$$


At the beginning, we need an initial guess for the QLM, so we considered as $g_{0}(\eta)=\eta$ which satisfies some of the boundary conditions of the problem. Mandelzweig and Tabakin [26] have shown that if the initial guess is true in one of the boundary conditions then the QLM is convergent.

We approximate the function $y(\eta)$ defined over a semiinfinite interval as follows:

$$
y(\eta)=\sum_{j=0}^{\infty} a_{j} R P_{j}(\eta)
$$

where $a_{j}$ is the unknown coefficient. By truncating Eq. (13) in the $\mathrm{N}$-th period, we have:

$$
y(\eta)=\sum_{j=0}^{N-1} a_{j} R P_{j}(\eta)=A(R P(\eta))^{T},
$$

where $A=\left[a_{0}, a_{1}, \cdots, a_{N-1}\right]$ and $R P(\eta)=$ $\left[R P_{0}(\eta), R P_{1}(\eta), \cdots, R P_{N-1}(\eta)\right]$. Due to the Eq. (14), $g_{i+1}(\eta)$ can be defined as follows:

$$
g_{i+1}(\eta)=\eta+\eta^{2} \sum_{j=0}^{N-1} a_{j} R P_{j}(\eta) .
$$

Eq. (15) satisfies the boundary conditions (Ritz method), other than $g{ }^{\prime+1}(\infty)=0$. We consider a sufficiently large number $M$ and suppose $g_{i+1}^{\prime}(M)=0$ to satisfy the last boundary condition. In this paper, we assumed $M$ is equal to the largest root of $R P_{N}(\eta)$ on the domain $(0, \infty)$. Eq. (15) is replaced in Eq. (11); so, the residual function is obtained.

$$
\begin{aligned}
\operatorname{Res}_{i+1}(\eta)= & -g_{i+1}^{\prime \prime \prime}(\eta)+G+\left(g_{i+1}(\eta)-g_{i}(\eta)\right) \frac{d G}{d g_{i}(\eta)} \\
& +\left(g_{i+1}^{\prime}(\eta)-g_{i}^{\prime}(\eta)\right) \frac{d G}{d g_{i}^{\prime}(\eta)} \\
& +\left(g_{i+1}^{\prime \prime}(\eta)-g_{i}^{\prime \prime}(\eta)\right) \frac{d G}{d g_{i}^{\prime \prime}(\eta)},
\end{aligned}
$$

where $G=\frac{\left(g_{i}^{\prime}(\eta)\right)^{2}-g_{i}(\eta) g_{i}^{\prime \prime}(\eta)}{\varepsilon+1-\varepsilon \delta\left(g_{i}^{\prime \prime}(\eta)\right)^{2}}$.

In this paper, the roots of $R P_{N}(\eta)$ are considered as the collocation points. By collocating the points in residual function, a system of $N$ linear algebraic equations is obtained. We approximated the function $g$ by solving this system.

\section{Numerical results and discussion}

In this part, the numerical simulation and the results are presented. Moreover, the effects of the non-dimensional fluid parameters on the derivatives of the dimensionless stream function are shown.

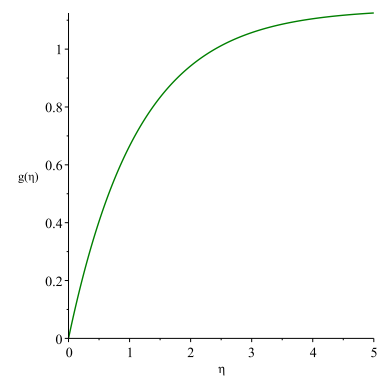

(a) $g(\eta)$

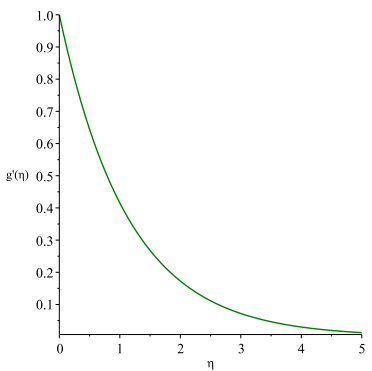

(b) $g^{\prime}(\eta)$

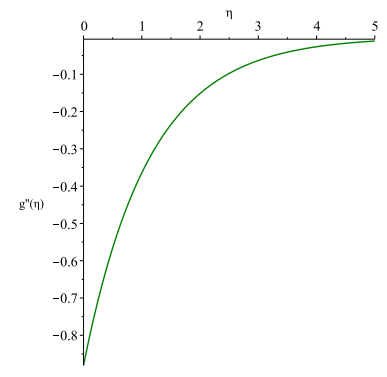

(c) $g^{\prime \prime}(\eta)$

Fig. 2: The $g(\eta), g^{\prime}(\eta), g^{\prime \prime}(\eta)$ functions for $N=60$ and iteration $=20$.

Tables 2 and 3 contain the values of $g^{\prime}(\eta)$ and $g^{\prime \prime}(\eta)$ for different values of $\eta$ by the different number of collocation points $(20,50$, and 60$)$ in the 20 -th iteration.

In Fig. 2, the graphs of the profile of the $g(\eta), g^{\prime}(\eta)$ and $g^{\prime \prime}(\eta)$ functions for various values of $\eta$ and $\varepsilon=0.3, L=10$ and $\delta=0.1$ are shown. An approximation of the optimal value of $L$ is calculated by the experimental trial-and-error method which is provided in page 377 from Ref. [25].

Fig. 3 demonstrates the logarithm residual errors for $N=20,50$ and 60 in the 20-th iteration. We can see that by increasing the number of collocation points, the values of residual errors are decreasing. Since the residual errors are very close to zero, it is obtained that the presented method has a high accuracy, and also the method is convergent and that means by increasing collocation points the values of residual errors tends to zero. 
Table 1: Obtained values of $g^{\prime}(\eta)$ for different values of $N$ for iteration $=20$ by RP functions.

\begin{tabular}{c|ccc}
\hline$\eta$ & $N=20$ & $N=50$ & $N=60$ \\
\hline 0 & 1 & 1 & 1 \\
0.2 & 0.83859898936489102 & 0.83859899444647251 & 0.83859899444647171 \\
0.4 & 0.70340102201207420 & 0.70340103213149533 & 0.70340103213149372 \\
0.6 & 0.59009171034899664 & 0.59009172560055394 & 0.59009172560055149 \\
0.8 & 0.49509069371157298 & 0.49509071475151664 & 0.49509071475151330 \\
1.0 & 0.41541764409164644 & 0.41541767112373892 & 0.41541767112373467 \\
1.2 & 0.34858612521610088 & 0.34858615807434099 & 0.34858615807433578 \\
1.4 & 0.29251833374407672 & 0.29251837242730295 & 0.29251837242729676 \\
1.6 & 0.24547586225692808 & 0.24547590708109875 & 0.24547590708109156 \\
1.8 & 0.20600299666190231 & 0.20600304802931573 & 0.20600304802930752 \\
2.0 & 0.17287997933504899 & 0.17288003745281602 & 0.17288003745280679 \\
2.2 & 0.14508429985423372 & 0.14508436462027636 & 0.14508436462026612 \\
2.4 & 0.12175851903351545 & 0.12175859010531514 & 0.12175859010530392 \\
2.6 & 0.10218345257016956 & 0.10218352952074103 & 0.10218352952072884 \\
2.8 & 0.85755778095000103 & 0.08575586055401851 & 0.08575586055400539 \\
3.0 & 0.07196930951371833 & 0.07196939723766221 & 0.07196939723764819 \\
3.2 & 0.06039932199907435 & 0.06039941486476310 & 0.06039941486474821 \\
3.4 & 0.05068942095398558 & 0.05068951890136260 & 0.05068951890134690 \\
3.6 & 0.04254053624782372 & 0.04254063920696544 & 0.04254063920694898 \\
3.8 & 0.03570169426103699 & 0.03570180209199399 & 0.03570180209197681 \\
4.0 & 0.02996227848139103 & 0.02996239094248002 & 0.02996239094246217 \\
4.2 & 0.02514553729838496 & 0.02514565404573913 & 0.02514565404572066 \\
4.4 & 0.02110313727666625 & 0.02110325788880624 & 0.02110325788878719 \\
4.6 & 0.01771059311270872 & 0.01771071712953130 & 0.01771071712951171 \\
4.8 & 0.01486343290797376 & 0.01486355987314963 & 0.01486355987312956 \\
5.0 & 0.01247398029174902 & 0.01247410978968198 & 0.01247410978966146 \\
\hline & & &
\end{tabular}

In Fig. 4, the effects of the value of $\varepsilon$ on $g^{\prime}(\eta), g^{\prime \prime}(\eta)$ with $\delta=0.1$ are shown.

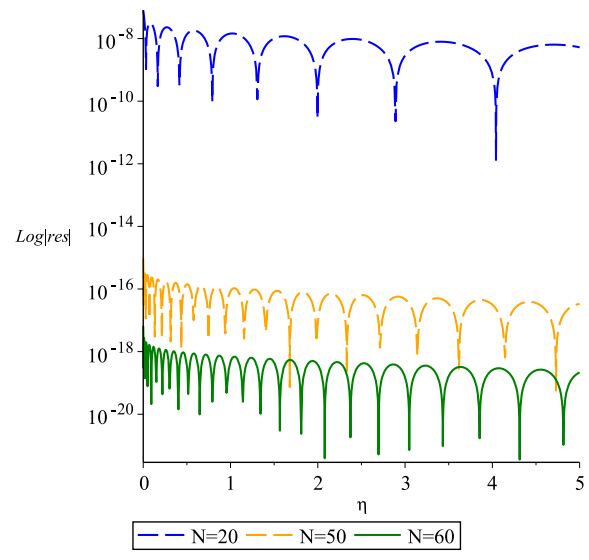

Fig. 3: The logarithm residual errors for RP for $N=20,50$, and 60 and iteration $=20$.

\section{Conclusion}

In this paper, the rational Legendre functions (RLFs) collocation method has been presented for solving a nonlinear ordinary differential equation in a semi-infinite interval. The explained problem is the boundary layer flow of a non-Newtonian fluid over a linear stretching sheet. The quasilinearization method is discussed to convert the equation to a sequence of linear differential equations. This sequence is solved by the rational Legendre collocation method. The residual errors and the results indicate the highly admitted accuracy and convergence of the proposed method. The present method has some advantages, for example:

- The effects of non-Newtonian fluid material parameters are investigated on the velocity and stress profiles of a non-Newtonian Eyring-Powell fluid over a linearly stretching sheet.

- $\quad$ The effects of Powell-Eyring fluid parameters on velocity and stress profiles are quite opposite.

- The roots of the RLFs were used in the interval $[0, \infty)$ as collocation points to solve the equation and do not reform the problem to a finite domain. 
Table 2: Obtained values of $g^{\prime \prime}(\eta)$ for different values of $N$ for iteration $=20$ by RP functions.

\begin{tabular}{c|ccc}
\hline$\eta$ & $N=20$ & $N=50$ & $N=60$ \\
\hline 0 & -0.88078499747125922 & -0.88078497244601874 & -0.88078497244602272 \\
0.2 & -0.73755704202254789 & -0.73755701571027815 & -0.73755701571028217 \\
0.4 & -0.618003787858089621 & -0.61800376335769558 & -0.61800376335769972 \\
0.6 & -0.518061915396319429 & -0.51806188800884449 & -0.51806188800884880 \\
0.8 & -0.434423093230810111 & -0.43442306325249482 & -0.43442306325249933 \\
1.0 & -0.364372384768658295 & -0.36437235516508404 & -0.36437235516508873 \\
1.2 & -0.305668577673621639 & -0.30566854883410831 & -0.30566854883411316 \\
1.4 & -0.256453314708564489 & -0.25645328499869334 & -0.25645328499869830 \\
1.6 & -0.215180623218004124 & -0.21518059144444689 & -0.21518059144445195 \\
1.8 & -0.180561289199228789 & -0.18056125571972253 & -0.18056125571972762 \\
2.0 & -0.151518308569813979 & -0.15151827481996751 & -0.15151827481997256 \\
2.2 & -0.127150795231806599 & -0.12715076270648465 & -0.12715076270648965 \\
2.4 & -0.106704476071187646 & -0.10670444560744341 & -0.10670444560744831 \\
2.6 & -0.089547404015357987 & -0.08954737563760644 & -0.08954737563761120 \\
2.8 & -0.075149862220013972 & -0.07514983540086583 & -0.07514983540087041 \\
3.0 & -0.063067671647562489 & -0.06306764571285020 & -0.06306764571285460 \\
3.2 & -0.052928285960463070 & -0.05292826042390378 & -0.05292826042390796 \\
3.4 & -0.044419184026325635 & -0.04441915875546242 & -0.04441915875546637 \\
3.6 & -0.037278165624955666 & -0.03727814083905698 & -0.03727814083906069 \\
3.8 & -0.031285229374198879 & -0.03128520552943696 & -0.03128520552944044 \\
4.0 & -0.026255769535789220 & -0.02625574716651940 & -0.02625574716652264 \\
4.2 & -0.022034874327473658 & -0.02203485389997206 & -0.02203485389997506 \\
4.4 & -0.018492545485008100 & -0.01849252729709350 & -0.01849252729709627 \\
4.6 & -0.015519689089803195 & -0.01551967322725212 & -0.01551967322725466 \\
4.8 & -0.013024752557373998 & -0.01302473890149214 & -0.01302473890149447 \\
5.0 & -0.010930903247790180 & -0.01093089151937285 & -0.01093089151937498 \\
\hline & & & \\
\hline & & & \\
\hline
\end{tabular}

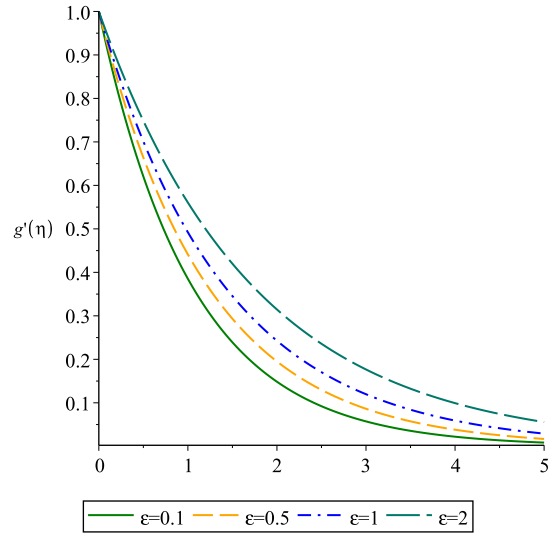

(a) The effect of $\varepsilon$ on $g^{\prime}(\eta)$.

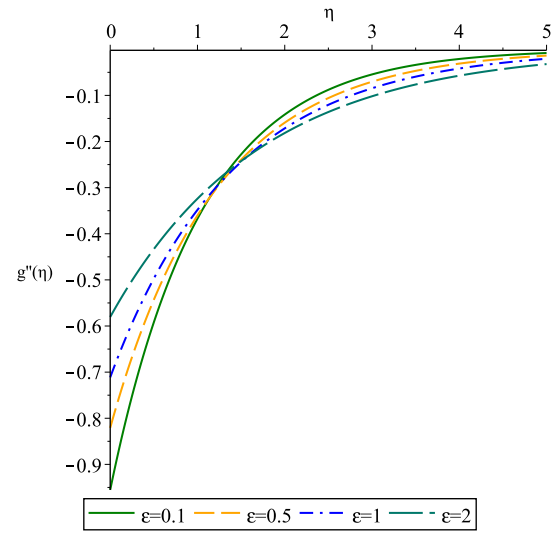

(b) The effect of $\varepsilon$ on $g^{\prime \prime}(\eta)$.

Fig. 4: The effects of the value of $\varepsilon$ on $g^{\prime}(\eta), g^{\prime \prime}(\eta)(\delta=0.1)$.

- $\quad$ An approximation of the optimal value of $L$ is calculated in the rational Legendre functions $R P_{n}^{\alpha}(x, L)$ to solve the equation.

- The convergence of the obtained results is shown with the reduction of the residual errors.

- An accurate solution for $g(\eta), g^{\prime}(\eta)$ and $g^{\prime \prime}(\eta)$ is obtained.
- This method is a good experience and method for the other sciences. 


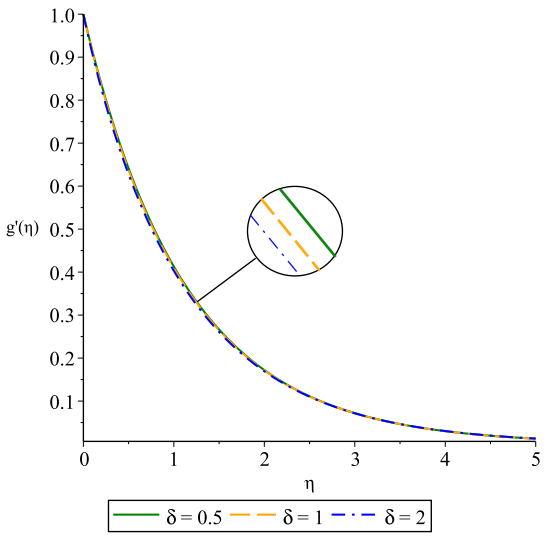

(a) The effect of $\delta$ on $g^{\prime}(\eta)$

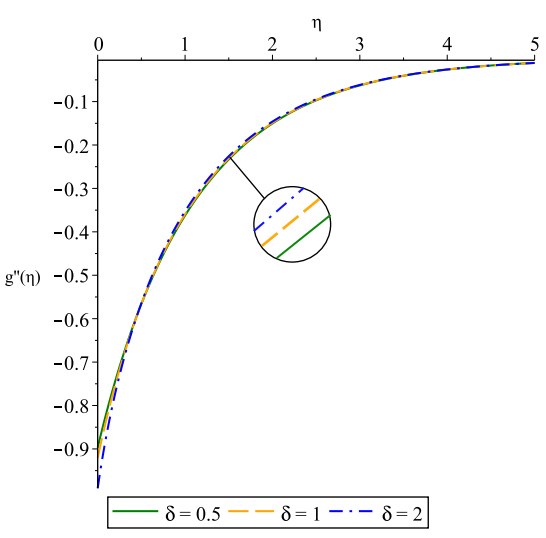

(b) The effect of $\delta$ on $g^{\prime \prime}(\eta)$.

Fig. 5: The effects of the value of $\delta$ on $g^{\prime}(\eta), g^{\prime \prime}(\eta)(\varepsilon=0.3)$.

\section{A The quasilinearization method}

Most algorithms for solving nonlinear equations or systems of equations are variations of Newton's method. When we apply the collocation method for solving a nonlinear differential equation, the result is a nonlinear system of algebraic equations. The aim of the quasilinearization method (QLM) pioneered by Bellman and Kalaba [27] for solving the $n$-order nonlinear differential equations in $N$ dimensions as the limit of a sequence of linear differential equations. The general properties of the method, particularly its uniform and quadratic convergence, which often also is monotonic [26]. The QLM is easily understandable since there is no difficult technique for obtaining the general solution of a nonlinear equation in terms of a finite set of particular solutions.

To know how the QLM works, imagine that we have one nonlinear ordinary differential equation in one variable on the interval $[0, b]$ as $[26,27]$ :

$$
L^{(n)} y(x)=f\left(y(x), y^{(1)}(x), \cdots, y^{(n-1)}(x), x\right),
$$

with $n$ boundary conditions

$$
\begin{aligned}
& g_{k}\left(y(0), y^{(1)}(0), \cdots, y^{(n-1)}(0)\right)=0, \quad k=1, \cdots, l \\
& g_{k}\left(y(b), y^{(1)}(b), \cdots, y^{(n-1)}(b)\right)=0, \quad k=l+1, \cdots, n,
\end{aligned}
$$

where $L^{(n)}$ is $n$-order linear ordinary differential operator and $f$ and $g_{1}, g_{2}, \cdots, g_{n}$ are nonlinear functions of $y(x)$ and its $(n+1)$ derivatives $y^{(s)}(x), s=1, \cdots, n$. The QLM determines the $(r+1)$ th iterative approximation $y_{r+1}(x)$ to the solution of Eq. (17) as a solution of the linear differen- tial equation:

$L^{(n)} y_{r+1}(x)=f\left(y_{r}(x), y_{r}^{(1)}(x), \cdots, y_{r}^{(n-1)}(x), x\right)$

$+\sum_{s=0}^{n-1}\left(y_{r+1}^{(s)}(x)-y_{r}^{(s)}(x)\right) f_{y^{(s)}}\left(y_{r}(x), y_{r}^{(1)}(x), \cdots, y_{r}^{(n-1)}(x), x\right)$,

where $y_{r}^{(0)}(x)=y_{r}(x)$, with linearized two-point boundary conditions:

$$
\begin{gathered}
\sum_{s=0}^{n-1}\left(y_{r+1}^{(s)}(0)-y_{r}^{(s)}(0)\right) g_{k y^{(s)}}\left(y_{r}(0), y_{r}^{(1)}(0), \cdots, y_{r}^{(n-1)}(0), 0\right), \\
k=1, \cdots, l
\end{gathered}
$$

and

$$
\begin{gathered}
\sum_{s=0}^{n-1}\left(y_{r+1}^{(s)}(b)-y_{r}^{(s)}(b)\right) g_{k y^{(s)}}\left(y_{r}(b), y_{r}^{(1)}(b), \cdots, y_{r}^{(n-1)}(b), b\right), \\
k=l+1, \cdots, n,
\end{gathered}
$$

where the functions $f_{y^{(s)}}=\frac{\partial f}{\partial y^{(s)}}$ and $g_{k y^{(s)}}=\frac{\partial g_{k}}{\partial y^{(s)}}$, $s=1,2, \cdots, n-1$ are functional derivatives of the functionals $f\left(y_{r}(x), y_{r}^{(1)}(x), \cdots, y_{r}^{(n-1)}(x), x\right)$ and $g_{k}\left(y_{r}(x), y_{r}^{(1)}(x), \cdots, y_{r}^{(n-1)}(x), x\right)$, respectively. The QLM iteration needs an initialization or initial guess $y_{0}(\eta)$ that it is chosen from physical and mathematical considerations or the boundary conditions in the equation.

\section{References}

[1] Bilal Ashraf M, Hayat T, Alsaedi A. Three-dimensional flow of Eyring-Powell nanofluid by convectively heated exponentially stretching sheet. Eur. Phys. J. Plus 2015130 5:1-16.

[2] Rahimi J, Ganji D, Khaki M, Hosseinzadeh K. Solution of the boundary layer flow of an Eyring-Powell non-Newtonian fluid 
over a linear stretching sheet by collocation method. Alex.

Eng. J. 201756 621-627.

[3] Javed T, Ali N, Abbas Z, Sajid M. Flow of an Eyring-Powell nonNewtonian fluid over a stretching sheet, Chem. Eng. Commun 2013200 327-336.

[4] Rehman KU, Ali Khan A, Malik MY. Magneto-nanofluid numerical modelling of chemically reactive Eyring-Powell fluid flow towards both flat and cylindrical an inclined surfaces. A comparative study. AlP Advances 20177065103.

[5] Crane L.J, Angew Z. Flow past a stretching plate. Math. Phys. 197021 645-647.

[6] Khan I, Qasim M, Shafie S. Flow of an Erying-Powell fluid over a stretching sheet in presence of chemical reaction. Thermal Sci. 201620 1903-1912.

[7] Harris J. Rheology and Non-Newtonian Flow, Longman 1977.

[8] Bird RB, Curtis CF, Armstrong RC, Hassager O. Dynamics of Polymeric Liquids. Volume 1 Fluid Mechanics, Wiley publisher 1987.

[9] Powell R. E, Eyring H, Mechanisms for the relaxation theory of viscosity. Nature 1944154 427-428.

[10] Hayat T, Aslam N, Rafiq M, Alsaadi FE. Hall and Joule heating effects on peristaltic flow of Powell-Eyring liquid in an inclined symmetric channel. Results Phys. 2017 518-528.

[11] Gaffar SA, Ramachandra Prasad V, Reddy EK. MHD free convection flow of Eyring-Powell fluid from vertical surface in porous media with Hall/ionslip currents and ohmic dissipation. Alex. Eng. J. 201655 875-905.

[12] Takhar HS, et al.. Flow and mass transfer on a stretching sheet with magnetic field and chemical reactive species. Int. J. Eng. Sci. 200038 1303-1314.

[13] Megahed AM. Flow and heat transfer of Powell-Eyring fluid due to an exponential stretching sheet with heat flux and variable thermal conductivity. Z. Naturforsch A 201570 163-169.

[14] Rehman KU, Saba NU, Malik MY, Malik AA. Encountering heat and mass transfer mechanisms simultaneously in PowellErying fluid through Lie symmetry approach. Nonlinear Studies 2017 10 541-549.

[15] Ellahi R, Shivanian E, Abbasbandy S, Hayat T. Numerical study of magnetohydrodynamics generalized Couette flow of EyringPowell fluid with heat transfer and slip condition. Int. J. Numer. Method. H. F. F. 201626 1433-1445.

[16] Khan NA, Sohail A, Sultan F. Effect of anisotropic slip and magnetic field on the flow and heat transfer of Eyring-Powell fluid over an infinite rotating disk. Int. J. Fluid Mech. Res. 201744 257-273.
[17] Khan NA, Sultan F, Rubbab Q. Optimal solution of nonlinear heat and mass transfer in a two-layer flow with nano-EyringPowell fluid. Results in Physics. 20155 199-205.

[18] Khan NA, Sultan F. On the double diffusive convection flow of Eyring-Powell fluid due to cone through a porous medium with Soret and Dufour effects. AIP advances. 20155057140.

[19] Parand K, Abbasbandy S, Kazem S, Rad J. A novel application of radial basis functions for solving a model of first-order integro-ordinary differential equation, Commun. Nonlinear Sci. Numer. Simul. 201116 4250-4258.

[20] Parand K, Delkhosh M. An accurate numerical method for solving unsteady isothermal flow of a gas through a semi-infinite porous medium. J. Comput. Nonlinear Dyn. 201813011007.

[21] Baharifard F, Kazem S, Parand K. Rational and exponential Legendre tau method on steady flow of a third grade fluid in a porous half space. Int. J. Appl. Comput. Math. 20162 679-698.

[22] Parand K, Delkhosh M. Solving the nonlinear Schlomilch's integral equation arising in ionospheric problems. Afr. Mat. 201728 459-480.

[23] Parand K, Mirahmadian F, Delkhosh M. The pseudospectral Legendre method for solving the HIV infection model of $\mathrm{CD4}^{+} \mathrm{T}$ cells. Nonlinear Studies 201825 241-250.

[24] Parand K, Delafkar Z, Amani R. J, Kazem S. Numerical study on wall temperature and surface heat flux natural convection equations arising in porous media by rational Legendre pseudo-spectral approach. Int. J. Nonlinear Sci. 20109 1-12.

[25] Boyd JP. Chebyshev and Fourier Spectral Methods. second ed., Dover, Mineola, New York. 2001.

[26] Mandelzweig V, Tabakin F. Quasilinearization approach to nonlinear problems in physics with application to nonlinear ODEs. Comput. Phys. Commun. 2001141 268-281.

[27] Kalaba RE, Bellman RE. On nonlinear differential equations, the maximum operation, and monotone convergence. J. Math. Mech. 19598 519-574.

[28] Parand K, Delkhosh M. Accurate solution of the Thomas-Fermi equation using the fractional order of rational Chebyshev functions. J. Comput. Appl. Math. 2017317 624-642.

[29] Parand K, Delkhosh M. An Efficient Numerical Solution of Nonlinear Hunter-Saxton Equation. Commun. Theor. Phys. 201767 483-492.

[30] Parand K, Yousefi H, Delkhosh M. A Numerical Approach to Solve Lane-Emden Type Equations by the Fractional Order of Rational Bernoulli Functions. Romanian J. Phys. 201762 104:124.

[31] Canuto C, Hussaini MY, Quarteroni A, Zang TA. Spectral Methods in Fluid Dynamic, Springer-Verlag, New York, 1987. 